\title{
Nocturnal eye movements in patients with idiopathic rapid eye movement sleep behaviour disorder and patients with Parkinson's disease
}

\author{
Christensen, Julie Anja Engelhard; Cesari, Matteo; Pizza, F.; Plazzi, G.; Jennum, P.
}

Published in:

Journal of Sleep Research

Publication date:

2018

Document Version

Publisher's PDF, also known as Version of record

Link back to DTU Orbit

Citation (APA):

Christensen, J. A. E., Cesari, M., Pizza, F., Plazzi, G., \& Jennum, P. (2018). Nocturnal eye movements in patients with idiopathic rapid eye movement sleep behaviour disorder and patients with Parkinson's disease. Journal of Sleep Research, 27(Suppl. 1, Sp. Iss. SI), [P209 ].

\section{General rights}

Copyright and moral rights for the publications made accessible in the public portal are retained by the authors and/or other copyright owners and it is a condition of accessing publications that users recognise and abide by the legal requirements associated with these rights.

- Users may download and print one copy of any publication from the public portal for the purpose of private study or research.

- You may not further distribute the material or use it for any profit-making activity or commercial gain

- You may freely distribute the URL identifying the publication in the public portal 
correlation between apnoeas and vagus stimulation. VNS settings during the recording were: an intensity of $2.25 \mathrm{mAmps}$, frequency of $30 \mathrm{HZ}$, pulse width 500 microseconds $(\mathrm{ms})$, on-time of $30 \mathrm{~s}$ and offtime of $180 \mathrm{~s}$. A lot of epileptic activity on the electroencephalography caused difficulties when defining sleep stages.

Results: PSG showed 22 central apnoeas and 36 central hypopneas. However the patient had additional central hypopneas consisting of episodes of decreased airflow without desaturations. Average $\mathrm{SaO} 2$ desaturation was $97 \%$ and the lowest $\mathrm{SaO} 2$ was $91 \%$. $\mathrm{AHI}$ was 11 , but hypopneas without desaturation were not included. Heart arrhythmias happened during breathing events.

Practically all of breathing events and heart arrhythmias were periodic and corresponded to VNS stimulation (every 3 minutes). They persisted for the duration of the stimulation (30 s) and came to an end with VNS deactivation.

Conclusions: VNS may cause central apnoeas corresponding to VNS stimulation. PSG should be considered in patients presenting with apnoeas and EDS and adjustment of VNS settings is required.

Disclosure: Nothing to disclose.

\section{P208 | The association between shift work and migraine, tension-type headache and medication- overuse headache - a cross-sectional study among a large population of nurses}

B. Bjorvatn ${ }^{1,2}$; S. Pallesen ${ }^{2,3}$; B.E. Moen ${ }^{1}$; S. Waage ${ }^{1,2}$; E.S. Kristoffersen ${ }^{4}$

${ }^{1}$ Department of Global Public Health and Primary Care, University of Bergen, ${ }^{2}$ Norwegian Competence Center for Sleep Disorders, Haukeland University Hospital, ${ }^{3}$ Department of Psychosocial Science, University of Bergen, Bergen, ${ }^{4}$ Department of General Practice, University of Oslo, Oslo, Norway

Objectives/Instruction: Shift work is associated with poor sleep, and poor sleep may trigger headache. However, whether there is an association between shift work and headache is unclear. The objective of this study was to investigate possible associations between different types of headache and shift work.

Methods: 1585 nurses with different work schedules (day work, twoshift rotation, night work, three-shift rotation) participated in a cohort study with annual surveys that started in 2008/2009. A comprehensive headache instrument was included in wave 6 . Headaches were assessed according to the International Classification of Headache Disorders IIlb. Frequent headache ( $\geq 1$ day per month), migraine, tension-type headache (TTH), chronic headache (headache $>14$ days per month), and medication-overuse headache $(\mathrm{MOH}$, chronic headache + acute headache medication $\geq 10$ days last month) comprised the dependent variables. Adjusted (for sex, age, percentage of full-time equivalent, marital status, children living at home) logistic regression analyses were conducted with work schedule, number of night shifts worked last year, number of quick returns ( $<11 \mathrm{hr}$ in-between shifts) last year, shift work disorder (SWD), and insomnia disorder as predictors.
Results: Frequent headache, migraine, and chronic headache were significantly associated with SWD (ORs 1.60 to 2.45) and insomnia disorder (ORs 1.55 to 3.03), but not with work schedule, number of night shifts or number of quick returns. TTH was only associated with $>20$ night shifts last year

(OR 1.41). $\mathrm{MOH}$ was only associated with insomnia disorder (OR 7.62).

Conclusions: We found no association between different types of headache and work schedule. However, TTH was associated with high number of night shifts. Nurses with insomnia disorder and SWD reported higher prevalence of frequent headache, migraine, chronic headache and $\mathrm{MOH}$ (only insomnia) compared to nurses not having insomnia disorder and SWD, respectively.

Disclosure: Nothing to disclose.

\section{P209 | Nocturnal eye movements in patients with idiopathic rapid eye movement sleep behaviour disorder and patients with Parkinson's disease}

J.A.E. Christensen ${ }^{1,2}$; M. Cesari ${ }^{1,2}$; F. Pizza ${ }^{3,4}$; G. Plazzi ${ }^{3,4}$; P. Jennum ${ }^{5}$

${ }^{1}$ Danish Center for Sleep Medicine, Department of Clinical Neurophysiology, Rigshospitalet, Glostrup, ${ }^{2}$ Department of Electrical Engineering, Technical University of Denmark, Kgs Lyngby, Denmark, ${ }^{3}$ Department of Biomedical and Neuromotor Sciences, University of Bologna, ${ }^{4}$ IRCCS Institute of the Neurological Sciences, AUSL di Bologna, Bologna, Italy, ${ }^{5}$ Department of Clinical Neurophysiology, Rigshospitalet, Danish Center for Sleep Medicine, Glostrup, Denmark

Objectives/Introduction: Patients with idiopathic rapid eye movement (REM) sleep behaviour disorder (iRBD) have a high risk of converting into $\alpha$-synucleinopathies due to an early neurodegenerative process involving lower brainstem areas. Eye movements (EMs) are controlled by neurons located in the lower brainstem, midbrain, and frontal areas, and are therefore vulnerable to be affected by an early neurodegeneration. Several studies have reported impairment of the oculomotor function in patients with Parkinson's disease (PD) during wakefulness, but no studies have investigated how neurodegeneration affects EMs during sleep. We aimed to evaluate nocturnal EMs in $\mathrm{iRBD}$ and PD hypothesizing that these patients present abnormal EMs during sleep.

Methods: A total of 28 patients with periodic leg movement disorder (PLMD; 15 males; $60.3 \pm 11.9$ years), 24 with iRBD (17 males; $63.0 \pm 10.1$ years), 23 with PD without RBD (PD-RBD; 14 males; $61.4 \pm 5.4$ years), 29 with $\mathrm{PD}$ and RBD (PD+RBD; 19 males; $63.3 \pm 5.1$ years), and 24 controls (11 males; $56.7 \pm 9.9$ years) were included. A previous validated EM detector was used to automatically detect periods with any kind of EMs in artefact-free epochs between lights off and on. The EM coverage was computed as the percentage of time containing EMs, during stages of wakefulness, N1, N2, N3, and REM sleep. Between-group comparisons were 
performed using Wilcoxon-Ranksum tests, and the Benjamini-Hochberg procedure with a false discovery rate of 0.1 was used to control for multiple testing.

Results: We found that PD+RBD had significant less EM coverage during wakefulness compared to controls $(p<0.001)$, and significantly higher EM coverage during N2 compared to controls ( $p=0.003$ ) and PLMD $(p=0.004)$. Furthermore, PD-RBD showed significantly higher EM coverage during N2 compared to controls $(p<0.001)$ and PLMD $(p=0.001)$. Same trends were observed between iRBD and controls in wakefulness and N2, but were not significant after correction for multiple testing.

Conclusions: Patients with PD+/-RBD reflect abnormal EM coverage during nocturnal wakefulness and N2, suggesting that the disorder is complex and affects various parts of the basal brain. The different profiles of EM coverage in iRBD and PD+/-RBD may mirror different stages of central nervous system involvement across disease progression, a finding to be confirmed by future longitudinal studies.

Disclosure: Nothing to disclose.

\section{P210 | Apathy and depression in Parkinson's disease with parasomnias: gender matters}

M. Ntafouli; P. Bargiotas; C. Bassetti

Sleep-Wake-Epilepsy Center, Department of Neurology, University Hospital Bern, Bern, Switzerland

Objectives/Instruction: Mood disturbances are common nonmotor symptoms in patients with Parkinson's Disease (PD). However, it remains unclear if these symptoms are related to the disease itself or to disease comorbidities. The aim of the study was to investigate whether the presence of parasomnias in patients with PD is associated with depression and apathy.

Methods: PD patients, who underwent subjective and objective sleep-wake assessments, were grouped according to the presence or not of (any) parasomnia. The diagnosis of parasomnias was based on clinical and polysomnographic data. Apathy was assessed using the 14-item Starkstein Apathy Scale (cut-off $\geq 14$ ). Depression was assessed using the Hamilton Depression Scale (cut-off $\geq 9$ ). The ESS, PDQ39, and MMSE were used to evaluate sleepiness, quality of life and cognitive impairment, respectively.

Results: The two groups were statistically comparable with respect to age, gender, years of PD, severity of PD (H\&Y), and motor score (UPDRS-III). Apathy prevalence in the group with parasomnias was $53.3 \%$ while in the control group without parasomnias was $33.3 \%$. PD patients with parasomnia had significantly higher apathy and depression scores than PD patients without parasomnia, attributed mainly to the differences in the female group. Among PD patients with parasomnias, females had significantly higher apathy scores than males but no other differences with respect to motor and nonmotor symptoms was found (data not shown). Gender was the only independent predictor of apathy, with women presenting a higher risk compared to men.

Conclusions: The presence of a parasomnia seems to increase the risk of apathy and depression in PD patients and especially in female individuals. Therefore, symptoms of apathy and depression should be taken into consideration in those patients even if they do not meet the diagnostic criteria for a mood disorder. Future studies should focus further on the relationship between gender and other mood features in PD patients with parasomnias.

Disclosure: Nothing to disclose.

\section{P211 | Cheyne-Stokes respiration and the outcome of acute stroke}

S.-Y. Lee ${ }^{1}$; Y. Kim ${ }^{1}$; S.H. Kim ${ }^{1}$; H.B. Shin ${ }^{2}$; E.Y. Roh ${ }^{3}$

${ }^{1}$ Kangwon National University School of Medicine, Chuncheon-si,

${ }^{2}$ Kosleep Sleep Clinic, ${ }^{3}$ Seoul National University Boramae Hospital, Seoul, Republic of Korea

Objectives/Instruction: Cheyne-Stokes respiration (CSR) is frequently observed in acute stroke patients. CSR has even been reported to be a poor prognostic factor of cardiac failure. However, the association between the presence of CSR and the outcome of acute stroke is not well established. We aimed to assess whether CSR has a predictive value in acute stroke.

Methods: We investigated the patients who were admitted with acute ischemic stroke and received nocturnal polysomnography. We collected data on demographics, risk factors, etiologic subtypes, and stroke outcome and the parameters associated with respiratory events in polysomnography. The stroke outcome was evaluated with the occurrence of early neurological deterioration (END) and modified Rankin Scale (mRS) at one and three months. We assessed the association between the presence of CSR and the stroke outcome using multivariate logistic regression analysis.

Results: Among 182 patients, 35 (19.2\%) showed CSR in polysomnography. Incidence of END was not different between groups according to CSR. MRS at one month and three months was higher in CSR group than non-CSR group. However, the association between CSR and mRS disappeared after adjustment by risk factors, severity and location of stroke using multivariate analysis, whereas the association between obstructive sleep apnea and higher mRS/ END persisted after adjustment.

Conclusions: The presence of CSR in acute stroke was associated with poor functional outcome. However, CSR was not an independent factor predicting the outcome of stroke.

Disclosure: Nothing to disclose. 